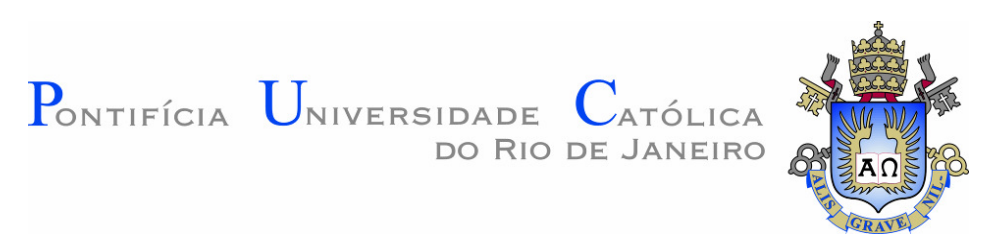

Oswaldo Ángel Francisco Robles Castillo

\title{
Análise Experimental do Escoamento de Emulsões Óleo em Água através de Micro-capilares com Garganta
}

\author{
Dissertação de Mestrado
}

Dissertação apresentada como requisito parcial para obtenção do título de Mestre pelo Programa de PósGraduação em Engenharia Mecânica da PUC - Rio.

Orientador: Prof. Márcio da Silveira Carvalho

Rio de Janeiro

Abril de 2011 


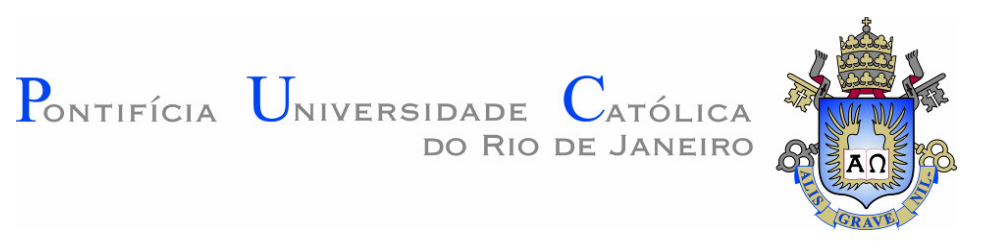

Oswaldo Ángel Francisco Robles Castillo

\title{
Análise Experimental do Escoamento de Emulsões Óleo em Água através de Micro-capilares com Garganta
}

Dissertação apresentada como requisito parcial para obtenção do título de Mestre pelo Programa de PósGraduação em Engenharia Mecânica da PUC-Rio. Aprovada pela Comissão Examinadora abaixo assinada.

\author{
Prof. Márcio da Silveira Carvalho \\ Orientador \\ Departamento de Engenharia Mecânica - PUC-Rio \\ Prof. Luis Fernando Alzuguir Azevedo \\ Departamento de Engenharia Mecânica - PUC-Rio \\ Prof. Paulo Roberto de Souza Mendes \\ Departamento de Engenharia Mecânica - PUC-Rio \\ Prof. José Eugenio Leal \\ Coordenador Setorial do Centro Técnico Científico - PUC-Rio
}


Todos os direitos reservados. É proibida a reprodução total ou parcial do trabalho sem autorização da universidade, do autor e do orientador.

\section{Oswaldo Ángel Francisco Robles Castillo}

Graduou-se em Engenharia Mecânica na Universidad Nacional de Trujillo - UNT (Trujillo, Perú) em 2004.

Ficha Catalográfica

Robles Castillo, Oswaldo Ángel Francisco

Análise experimental do escoamento de emulsões óleo em água através de micro-capilares com garganta / Oswaldo Ángel Francisco Robles Castillo ; orientador: Marcio da Silveira Carvalho. - 2011.

105 f. : il. (color.) ; $30 \mathrm{~cm}$

Dissertação (mestrado)-Pontifícia Universidade Católica do Rio de Janeiro, Departamento de Engenharia Mecânica, 2011.

Inclui bibliografia

1. Engenharia mecânica - Teses. 2. Emulsões. 3. Micro-capilares. 4. $\mu$-PIV. 5. Recuperação avançada de petróleo. 6. Meios porosos. I. Carvalho, Marcio da Silveira. II. Pontifícia Universidade Católica do Rio de Janeiro. Departamento de Engenharia Mecânica. III. Título.

CDD: 621 
Dedico este trabalho aos meus pais e irmãs, e em especial à Yngrid. 


\section{Agradecimentos}

A Deus pela sua companhia.

Ao meu orientador, professor Márcio da Silveira Carvalho, pelos ensinamentos, dedicação, apoio e confiança em mim prestada.

Ao Departamento de Engenharia Mecânica da Pontifícia Universidade Católica de Rio de Janeiro.

Ao $\mathrm{CNPq}$ pelo auxilio financeiro concedido, indispensável na realização deste trabalho.

Aos membros da banca examinadora, que aceitaram revisar o trabalho e contribuíram com valiosas observações.

Aos amigos e colegas do grupo de trabalho do professor Márcio Carvalho e do LMMP pela sua ajuda direta ou indireta na realização deste trabalho. 


\section{Resumo}

Robles Castillo, Oswaldo Ángel Francisco; Carvalho, Márcio da Silveira. Análise Experimental do Escoamento de Emulsões Óleo em Água através de Micro-capilares com Garganta. Rio de Janeiro, 2011. 105p. Dissertação de Mestrado - Departamento de Engenharia Mecânica, Pontifícia Universidade Católica do Rio de Janeiro.

No método de injeção de água, o óleo no reservatório é varrido até os poços produtores através de frentes de deslocamento não uniformes, deixando óleo estagnado em grandes regiões do reservatório. Frentes uniformes de deslocamento e uma melhor varredura do reservatório podem ser obtidas diminuindo a razão de mobilidade entre a água e o óleo. Normalmente, esta diminuição é feita através da modificação da razão de viscosidade entre ambas as fases. No método de injeção de emulsões, o controle da mobilidade é alcançado bloqueando os poros ou caminhos gerados pela água com gotas da fase dispersa com diâmetro da mesma ordem ou maior do que o tamanho de poro. A aplicação de emulsões no controle da mobilidade e o efeito do bloqueio de poro podem ser desenvolvidos mediante a análise de diferentes regimes de escoamento de emulsões em meios porosos. Neste trabalho, o estudo do escoamento de emulsões em meios porosos foi realizado mediante duas abordagens experimentais utilizando um micro-capilar com garganta para modelar uma garganta conectando dois poros adjacentes. $\mathrm{Na}$

primeira abordagem experimental, quantificou-se a queda de pressão para diferentes vazões com emulsões de três tamanhos de gota e duas concentrações de óleo em dois capilares diferentes. Os resultados confirmam que a razão entre o diâmetro da garganta do capilar e o tamanho de gota influencia fortemente a relação vazão-queda de pressão. Os resultados mostram que, para baixos números de capilaridade, o escoamento de emulsões é dominado por efeitos capilares e leva a uma diminuição da mobilidade local. Na segunda montagem experimental, o sistema de micro-velocimetria por imagem de partículas ou $\mu$-PIV foi utilizado para medir campos de velocidade do escoamento através de micro-capilares com garganta. Resoluções espaciais da ordem de $20 \mu \mathrm{m}$ foram obtidas para o campo de 
velocidade calculado pela média amostral de vários campos instantâneos de velocidade. Os resultados experimentais da relação vazão-queda de pressão e os campos de velocidade obtidos mediante o $\mu$-PIV representam informação de grande valor para o desenvolvimento de modelos de redes de capilares no estudo do escoamento de emulsões em meios porosos.

\section{Palavras-chave}

Emulsões; Micro-Capilares; $\mu$-PIV; Recuperação Avançada de Petróleo; Meios Porosos. 


\section{Abstract}

Robles Castillo, Oswaldo Ángel Francisco; Carvalho, Márcio da Silveira (Advisor). Experimental Analysis of the Oil-in-water Emulsion Flow through Constricted Micro-capillaries. Rio de Janeiro 2011. 105p. M.Sc. Dissertation - Departamento de Engenharia Mecânica, Pontifícia Universidade de Rio de Janeiro.

During water injection, oil is swept through the reservoir to production wells by non-uniform displacement fronts originating large areas of entrapped oil in the reservoir. Uniform displacement fronts and better reservoir sweep can be achieved by improving the mobility ratio between water and oil. Usually, mobility ratio is reduced by changing the viscosity ratio between both phases. When injecting emulsions, mobility control is achieved by blocking water paths with dispersed phase drops with diameter of the same order of magnitude of the pore throats size. The application of emulsions as mobility control agents and the pore blocking effect may be developed by analyzing different flow regimes of emulsions through porous media. In the analysis presented here, two experimental setups were used using a constricted quartz capillary to represent a pore throat that connects two adjacent pore bodies to study the flow of emulsions in the pore scale. In the first experiment, pressure drop was measured at different imposed volumetric flow rates for three oil drop size emulsions at two oil concentrations and two different quartz capillaries. The results show that the ratio between the capillary constriction diameter and the oil drop size has a strong influence on the flow rate-pressure drop relation. Experimental results also indicate that the emulsion flow dominated by capillary effects (low capillary number) leads to a decrease of local mobility. In the second experiment, a microscopic particle image velocimetry ( $\mu$-PIV) system was used to measure velocity fields of the flow of emulsion through a constricted micro-capillary. Ensemble-average was used in order to obtain resolution in the order of $20 \mu \mathrm{m}$. The flow rate-pressure drop relation results and the $\mu$-PIV velocity fields of the emulsion flow through a constricted micro-capillary represent invaluable information that can be used in 
the development of a capillary network model to study the flow of emulsions through porous media.

\section{Keywords}

Emulsions; Micro-Capillaries; $\mu$-PIV; Enhanced Oil Recovery; Porous Media. 


\section{Sumário}

1. Introdução 19

1.1. A produção de petróleo 21

1.2. Motivação 25

1.3. Objetivos 27

1.4. Escopo 28

2. Modelo simplificado de escoamento bifásico em um micro-capilar com garganta 29

2.1. Conceitos fundamentais 29

2.1.1. Emulsões 29

2.1.2. Estabilidade das emulsões 30

2.1.2.1. Surfactante 31

2.1.2.2. Concentração micelar crítica 32

2.1.3. Reologia das emulsões 33

2.1.4. Tensão superficial e tensão interfacial 33

2.1.5. Número de capilaridade $\quad 34$

2.2. Escoamento de emulsões através de micro-capilares 34

2.2.1. O fator de bloqueio 34

2.2.2. Mecanismos de bloqueio 36

2.2.2.1. Mecanismo Viscoso 36

2.2.2.2. Mecanismo Capilar 38

2.3. Revisão bibliográfica 40

3. Medição do fator de bloqueio 43

3.1. Abordagem experimental 43

3.1.1. Introdução 43

3.1.2. Formulação e preparação das emulsões 43

3.1.2.1. Preparação das fases 44

3.1.2.2. Caracterização das fases 46 
3.1.2.3. Preparação das emulsões

3.1.3. Caracterização da distribuição do tamanho de gota das emulsões 51

3.1.4. Bancada experimental 54

3.1.4.1. Sistema de Injeção 54

3.1.4.2. Sistema de medição da queda de pressão 56

3.1.4.3. Sistema de visualização do escoamento 58

3.1.5. Procedimento experimental 58

3.1.6. Dificuldades encontradas no processo experimental 60

3.2. Resultados 61

3.2.1. Efeito da geometria do capilar 65

3.2.2. Efeito da concentração de óleo nas emulsões 66

3.2.3. Efeito do tamanho de gota da fase dispersa 67

3.2.4. Fator de bloqueio em função do número de capilaridade $\quad 67$

$\begin{array}{ll}\text { 3.3. Comentários } & 71\end{array}$

4. Medição de campo de velocidade no escoamento bifásico através de um micro-capilar com garganta 73

4.1. Introdução 73

4.2. Abordagem experimental 76

4.2.1. Escolha dos fluidos de trabalho 76

4.2.2. Bancada experimental 77

4.2.2.1. Sistema de injeção

4.2.2.2. Sistema de micro-velocimetria por imagem de partículas $\quad 79$

4.2.3. Procedimento experimental 83

4.2.4. Dificuldades encontradas no processo experimental 85

4.3. Resultados 86

4.3.1. Campo de velocidade no escoamento da fase aquosa através de um micro-capilar com garganta 87

4.3.1.1. Escoamento da fase aquosa através da seção reta do micro-capilar com garganta 87

4.3.1.2. Escoamento da fase aquosa através da seção convergente da garganta do micro-capilar 91 
4.3.2. Campo de velocidade no escoamento bifásico através de um micro-capilar

4.4. Comentários

98

5. Comentários finais

100

Referências bibliográficas

102

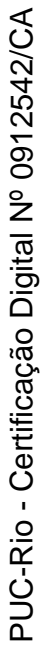




\section{Lista de figuras}

Figura 1.1: Consumo de energia mundial por tipo de combustível, 1990-2035 (Quatrilhão BTU).

Figura 1.2: Estrutura esquemática de um reservatório de petróleo. 22

Figura 1.3: Esquema de método miscível utilizando $\mathrm{CO}_{2}$ como solvente.(a) Deslocamento ideal. (b) Deslocamento com influência das densidades dos fluidos.

(c) Deslocamento com influência da alta diferença de viscosidades.

Figura 1.4: Esquema de método de injeção micelar de polímero.

Figura 1.5: Representação esquemática do método de recuperação de petróleo mediante injeção de água.

Figura 2.1: Tipos de emulsão segundo os seus componentes: emulsão água em óleo, emulsão óleo em água e emulsão múltipla a/o/a.

Figura 2.2: (A) Esquema simplificado de uma molécula ativa de superfície. (B) Estrutura molecular do surfactante dodecil sulfato de sódio.

Figura 2.3: Estrutura e orientação das moléculas de surfactante.

Figura 2.4: Processo de formação de micelas de surfactante.

Figura 2.5: Esquema do escoamento de uma gota de fase dispersa através de um capilar reto.

Figura 2.6: Perfis de velocidade do escoamento bifásico óleo-água através de um capilar reto de $100 \mu \mathrm{m}$ de diâmetro para uma queda de pressão de $5 \mathrm{kPa}$.

Figura 2.7: Esquema do escoamento de uma gota de fase dispersa através da garganta do capilar. 
Figura 3.1: Balança e surfactante utilizados na preparação das fases. 45

Figura 3.2: Dispensador de glicerina e agitador magnético usados no processo de homogeneização da fase contínua.

Figura 3.3: Bomba de vácuo e filtros empregados nos processos de filtração.

Figura 3.4: Balança e picnômetro utilizados na caracterização das fases.

Figura 3.5: Tensiômetro LAUDA VO 2001 e anel utilizado na medição da tensão superficial da fase contínua.

Figura 3.6: Pipeta de volume ajustável usada na preparação das emulsões.

Figura 3.7: Misturador mecânico rotativo Ultra Turrax T-25 e dispersor rotor-estator.

Figura 3.8: Componentes do analisador de partículas Mastersizer 2000.

Figura 3.9: Principio de determinação da distribuição do tamanho de partículas por difração laser. 51

Figura 3.10: Sistema Mastersizer 2000 e Hydro 2000MU. 52

Figura 3.11: Distribuição de tamanho de gota das emulsões de $5 \%$ em volume de óleo.

Figura 3.12: Distribuição de tamanho de gota das emulsões de $15 \%$ em volume de óleo.

Figura 3.13: Esquema da bancada experimental.

Figura 3.14: (a) Seringa, torneira três vias e capilar utilizados na injeção das emulsões. (b) Montagem dos componentes na bomba de seringa.

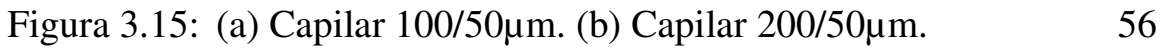

Figura 3.16: Transdutor de pressão Validyne DP15-TL. 56

Figura 3.17: Placa de terminais Validyne P/N 12871-1 e placa de interface Validyne UPC2100.

Figura 3.18: Cabo P/N 12870-5 utilizado na conexão entre as placas. $\quad 57$

Figura 3.19: Microscópio invertido Carl Zeiss Axiovert 40MAT. 58

Figura 3.20: Montagem do sistema de injeção e transdutor. 59 
Figura 3.21: Montagem do sistema de injeção e transdutor no microscópio.

Figura 3.22: Roteiro seguido no desenvolvimento da presente abordagem experimental.

Figura 3.23: Queda de pressão em função da vazão no escoamento das emulsões de $5 \%$ de óleo através do capilar $100 / 50 \mu \mathrm{m}$.

Figura 3.24: Queda de pressão em função da vazão no escoamento das emulsões de $5 \%$ de óleo através do capilar

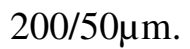

Figura 3.25: Queda de pressão em função da vazão no escoamento das emulsões de $15 \%$ de óleo através do capilar $200 / 50 \mu \mathrm{m}$.

Figura 3.26: Valores médios de queda de pressão em função da vazão no escoamento das emulsões de $5 \%$ de óleo

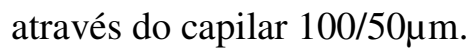

Figura 3.27: Valores médios de queda de pressão em função da vazão no escoamento das emulsões de $5 \%$ de óleo através do capilar 200/50 $\mu \mathrm{m}$.

Figura 3.28: Valores médios de queda de pressão em função da vazão no escoamento das emulsões de $15 \%$ de óleo através do capilar $200 / 50 \mu \mathrm{m}$.

Figura 3.29: Fator de bloqueio em função do número de capilaridade no escoamento das emulsões de 5\% de óleo através do capilar 100/50 $\mu \mathrm{m}$.

Figura 3.30: Fator de bloqueio em função do número de capilaridade no escoamento das emulsões de $5 \%$ de óleo através do capilar 200/50 $\mu$ m.

Figura 3.31: Fator de bloqueio em função do número de capilaridade no escoamento das emulsões de $15 \%$ de óleo através do capilar 200/50 $\mu \mathrm{m}$. 
Figura 4.1: Esquema da bancada experimental do sistema $\mu$-PIV.

Figura 4.2: Seringa de vidro de $100 \mu 1$ utilizada na injeção

da fase dispersa. $\quad 78$

Figura 4.3: Microscópio de fluorescência refletido Olympus ${ }^{\circledR}$. $\quad 79$

Figura 4.4: Objetiva Olympus 10x0.30 e cubo de filtro. 80

Figura 4.5: ${\text { Micro-esferas de poliestireno FluoSpheres }{ }^{\circledR} \text {. }}^{\circledR} \quad 80$

Figura 4.6: Fonte de energia e cabeça do laser Nd:YAG SOLO III. 81

Figura 4.7: Câmera CCD POWERVIEW ${ }^{\mathrm{TM}}$ de 1.4MP Mod. Sensicam-630066. 82

Figura 4.8: Sincronizador LaserPulse - Mod. 610034.

Figura 4.9: Montagem do sistema de injeção. 84

Figura 4.10: Montagem do sistema de injeção no microscópio. 84

Figura 4.11: Campo de vetores velocidade do escoamento através da seção reta obtido do processamento das imagens no software INSIGHT $3 \mathrm{G}^{\mathrm{TM}}$. 88

Figura 4.12: Campo de velocidade do escoamento da fase aquosa através da seção reta do micro-capilar.

Figura 4.13: Campo de velocidade do escoamento através da seção reta do micro-capilar apresentando $50 \%$ dos perfis de velocidade.

Figura 4.14: Comparação dos perfis parabólico e experimental.

Figura 4.15: Campo de vetores velocidade do escoamento através da seção convergente obtido do processamento das imagens no software INSIGHT $3 \mathrm{G}^{\mathrm{TM}}$.

Figura 4.16: Campo de velocidade do escoamento da fase aquosa através da seção convergente do micro-capilar.

Figura 4.17: Campo de velocidade do escoamento através da seção convergente do micro-capilar apresentando $50 \%$ dos perfis de velocidade.

Figura 4.18: Campo de velocidades obtido no estudo do escoamento através de um micro-bocal, desenvolvido por Wereley. 
Figura 4.19: Comparação dos perfis parabólicos e experimentais

na entrada e saída da seção convergente

do micro-capilar.

Figura 4.20: Variação da velocidade máxima do escoamento da fase aquosa na seção convergente do micro-capilar. 95

Figura 4.21: Variação da velocidade máxima média do escoamento da fase aquosa na seção convergente do micro-capilar. 95

Figura 4.22: Campo de velocidade do escoamento bifásico através da seção reta do micro-capilar. 


\section{Lista de tabelas}

Tabela 2.1: Macro-emulsões versus micro-emulsões.

Tabela 3.1: Propriedades das fases contínua e dispersa das emulsões a $23^{\circ} \mathrm{C}$.

Tabela 3.2: Parâmetros considerados no processo de emulsificação. 50

Tabela 3.3: Parâmetros de emulsificação e características morfológicas das emulsões. 